\title{
Structural Flexibility and Space Articulation in Architectural Design Teaching
}

\author{
Marina Ferreira Borges \\ UFMG | Brasil | marinafborges@gmail.com
}

\begin{abstract}
The separation between architectural design teaching and structural education corroborates the division of labor in professional practice that cannot support the development of dialectical relations between architects and engineers. Thus, the proposal of hybridization between architectural design teaching and structural education developed in this article presupposes a shift from the centrality given to the plastic and spatial principles of the architectural form to the development of approaches that are oriented towards the recognition of the material and constructive questions which aided by the parametric and structural behavior simulation tools allow the development of complex relationships based on tectonic procedural logic.
\end{abstract}

Keywords: Architectural design teaching; Structural education; Parametric design; Performance-based design.

\begin{abstract}
INTRODUCTION
Modern science and the concept of disciplinary teaching cannot cover all the complexity necessary for the practice of design, involving principles of spatiality, materiality and tectonics, requiring a transformation of teaching methods. This transformation requires an interdisciplinary articulation between architecture and engineering, stimulating a review of the disciplinary boundaries between the two fields through the construction of new design methods. Thus, the objective of this work was to experiment with possibilities of synthesis of architectural design through the architectural design teaching in conjunction with structural education, testing integration procedures via digital tools and their theoretical-conceptual design possibilities, thus seeking to overcome the difficulties of learning resulting from the current fragmentation of teaching, seeking to positively interfere in effective practice.
\end{abstract}

The initial hypothesis was that the digital design and structural analysis tools could offer an opportunity for integrating knowledge, through the possibilities of interactive and iterative assessment in the designed way, in such a way that the student develops skills to conceive and analyze structural systems computationally in an iterative way, bringing the project's relationship closer to the issues related to its spatial articulation, materiality and construction. In this way, the digital tools incorporated into the design process present a potential for the reconstruction of the current model through the development of non-conservative practices, enabling a common basis for the development of dialogical and dialectical relations between architects and the other agents of construction.

Thus, the proposal of hybridization in the teaching of projects carried out in this experiment by offering a discipline that aggregates parametric structural analysis presupposes a shift from the centrality given to the plastic and spatial principles of the architectural form to the development of approaches oriented to the recognition of material and constructive issues. Therefore, in order to make the experiments possible, it was necessary to partially unlock the technical code for structural engineering by learning computational methods of structural analysis, in such a way that access to information evaluating structural behavior could be achieved and incorporated as information for an architectural synthesis.

As an initial hypothesis, these relationships would occur through a man-machine conversation, in which architecture students would explore the possibilities of visualization, interaction and iteration with structural analysis software. However, this possibility expanded over the previous experiments to a man-machine-man conversation, a process in which structural analysis software became a common language among architecture students and engineers for the selection of structural parameters that informed the design synthesis.

The conception of the conversation that we worked as a methodological instrument for the construction of this pedagogical experiment was based mainly on the studies of reflective professional practices by the philosopher Donald Schön (1987) and in the construction and structuring of the conversation applied to design process carried out by cyberneticists Gordon Pask (1976), Heinz von Foerster (2003), Paul Pangaro and Hugh Dubberly (2009), in which it is defined that for the conversation to take place between two or more participants it is necessary to have a context, a common language, an agreement and an engagement, for so that actions and (trans) actions can take place between the participants

For Schön (1987), architectural design is a reflexive conversation, a representation of something to be brought to reality, involving complexity and synthesis, and demanding from its practitioners, skills to manage situations of uncertainty, instability and conflicts of interest. values. Thus, using the structure of cybernetic conversation and Schön's epistemological approach, we have structured a pedagogical methodology for the construction of the discipline whose structure and results we will show below. 


\section{METHODOLOGY}

\section{CONTEXT AND LANGUAGE}

The architectural design discipline Structural Flexibility and Spatial Articulation was a pedagogical experiment that consisted of an interface between design, structural theory and computational structural analysis. The objective was to experiment with research strategies on the convergence between flexibility and spatial articulation, problematizing as spatial decisions in conjunction with creative decisions, all of them related to the scarcity of context resources. With reference to the pedagogical experiments carried out by Black and Duff (1994) at the University of Berkeley and by Kara and Georgoulias (2012) in partnership between Harvard University and the Massachusetts Institute of Technology, it was proposed as a language for the practice of project was proposed to conduct a man-machine-man conversation through the combination of experimental methods (prototype design and tectonic approach looking for context analysis), computational methods (parametric modeling in Grasshopper and structural analysis in Karamba in plug-in) and analytical method (to learn and evaluate the conversion of the results found in the computational structural analysis).

The discipline was offered in the 2nd semester of 2018 as a proposal for experimentation carried out for research of a doctoral thesis. This was the seventh pedagogical experiment carried out in the research, having been developed as a test of the hypotheses raised in the previous experiments. The course was conducted by a project teacher, with the participation of two more project teachers in orientation and evaluation process, and a structural engineer, with 15 (fifteen) undergraduate students enrolled and one graduate student.

The curriculum of the architecture school in which the experiments were carried out has a certain flexibility, allowing professors in the Project Department to propose themes prior to enrollment, allowing students from the 3rd to the 10th term to enroll according to the affinity with the proposal made by the teacher. Thus, due to the different levels of knowledge of the enrolled students, the discipline was unable to work with subjects that required advanced knowledge of structural analysis, with a concern regarding the leveling of instrumental knowledge.

\section{AGREEMENT AND ENGAGEMENT}

Based on the parameters of the KYM Field Schools architectural design contest, the subject proposed by the discipline was the development of a project for schools in Africa through a parametric approach to the design process aided by the parametric and structural modeling of the software. analyze. For this purpose, the discipline was composed of theoretical modules with lectures, expository seminars and practical modules that consisted of guidelines, prototyping and software learning.

The theorical classes sought to provide students with concepts related to the design process and structural design procedures. After the inaugural class, the first class consisted of a procedural theoretical approach for the development of spatial articulation through a parametric project. To this end, 14 (fourteen) similar case studies of school design and construction in Africa were presented, in which the related socio-cultural, technical and constructive parameters were evidenced, seeking to encourage students to reproduce the parametric process.

The second theoretical class consisted of a review (or presentation) of basic structural concepts in order to enable students to carry out a structural analysis model. For this purpose, the concepts of Modulus of Elasticity (E), Shear Modulus (G), Coefficient of Thermal Expansion (A), Specific Weight ( $\mathrm{Y}$ ), Yield and Failure Limit Stresses (Fu) were seen. The types of actions on the structures were also addressed qualitatively, as well as the relationship between the applied actions (compression, traction, cutting, bending moment and torsion) and the resulting stresses (normal compression, normal stress, buckling, shearing), flexion and torsion). Finally, the relationships between active efforts and resistant sections were seen to assist students in choosing cross sections.

In this way, according to the concepts covered in the theoretical classes, students should present "Seminar on Structural Flexibility and Spatial Articulation", in which each group, composed of 3 (three) students, should research the characteristics of a given African country, such as the type of climate, economic, socio-cultural characteristics and vernacular construction solutions, to serve as parameters for the articulation of space and the development of structural systems best suited to architectural design. The definition of the country that each group would search for was carried out by lottery, in which the following options were placed: Senegal, Burkina Faso, Niger, Chad, RCA (Central African Republic), Sudan, Ethiopia, Uganda, Kenya and Somalia. The proposal was to promote the greatest possible diversification of proposals, due to the geographical distribution of the location.

After the general presentation of the parameters, each student should develop a school architectural project for a specific country and a specific climate, applying the selected parameters of spatial articulation and structural flexibility. For that, a physical prototype should be developed for study, serving as a reference for the later stage of modeling the structure in the parametric Grasshopper software and analysis in the parametric structural analysis Karamba plug-in.

To familiarize themselves with the computational parametric process, 3 (three) practical classes were offered for teaching Grasshopper, in such a way that the exercises developed corroborate the modeling of the structures. The students learned to model, in a sequence of complexity, beams, frames, flat trusses and space trusses. These models served as input for the learning classes of the structural analysis program Karamba, in which the approach was also carried out in a gradation of complexity.

The 4 (four) classes dedicated to parametric structural analysis consisted of instructions for assembling the model through the concept of structural design (REBELLO, 2000) and the input procedures required by the software. To practice the concepts and procedures learned, students performed structural analysis exercises, in which the results found in the computational analysis should converge to the solutions found by the analytical method. These exercises were performed for beams, gantries, flat trusses and spatial trusses, similarly reproducing the exercises performed in theoretical disciplines offered by the Structural Department. 
After becoming familiar with structural analysis through exercises, a conversation was initiated with the structural engineer, who made a presentation describing his training path to date and the experiences in professional practice in which he developed projects in a cooperative manner at architectural offices, envisioning a possibility of interdisciplinary practice. The students asked questions in order to understand how these cooperative processes are occurring, and how the roles of architects and engineers are defined in these practices. The engineer showed that in Brazil he had not yet had any similar experience, but that his experiences of training and professional practice abroad allowed him to understand the importance of the cooperation processes between architects and engineers in the design practice.

In the following classes, students held individual conversations with the engineer, and 2 (two) classes were held for this purpose. The first, which occurred at the beginning of the modeling process, consisted of an instrumental aid for configuring the model. The second, held two weeks later, consisted of a conversation to find structural solutions in their aesthetic and constructive aspects that would reconcile the intended spatiality with the structural efficiency criteria. The students were engaged with the circularity and recursion provided by the process, and after the initial difficulties of assembling and analyzing the model, they began to explore possibilities of form and resistance by changing the parameters. The students requested that the discipline be extended for another week so that there could be a recursion of the computational structural analysis process with the intended spatiality. This "return" to architectural considerations was accomplished through a conversation with an invited design teacher. The orientation for the final presentation of the project was that the selected spatial and structural parameters were explicitly evident in the proposal. The focus of the final presentation should be procedural, since the products obtained were the result of a negotiation of the parameters involved.

\section{ACTION AND (TRANS) ACTION}

The parameters for the design were defined by groups at the time of the seminar. The individual projects were based on the sociocultural and environmental characteristics identified in the countries studied, with each student following their own path for the realization of the project, selecting, among the parameters identified by the group, those they considered most relevant for the individual proposals. To illustrate the design process carried out by students, we will see below two examples made by students who developed proposals for Senegal.

To develop the architectural design, Senegal group identified climate, culture and construction typologies as fundamental parameters. The region's climate is characterized by a prolonged dry season and irregular and poorly distributed rains, which are divided into a semiarid and desert climate in the north and a dry tropical climate in the south. With regard to social organization, the tribes are characterized by loyalty to the leader, social and community life organized by age group and by education taking place in community houses, using traditional dances as a form of recreation. With regard to buildings, the characteristics are divided between those that are consolidated in an urban environment (definitive character, usually in reinforced concrete), those that are not consolidated (provisional character, with the use of adobe brick, cement or ceramic), and traditional buildings characterized by ethnic variety, using the materials of the region (wood, bamboo, stones and adobe).

Project A was carried out for construction in the African semi-arid region and consisted of a module that could be combined with different joints (Figure 1). The process was guided by the low availability of industrialized materials, having chosen only natural materials found in the place, such as wood, earth and straw. With regard to the design of the structure, an inclined porch was designed, in such a way that it provided a variation in the ceiling height, contributing to the air circulation.

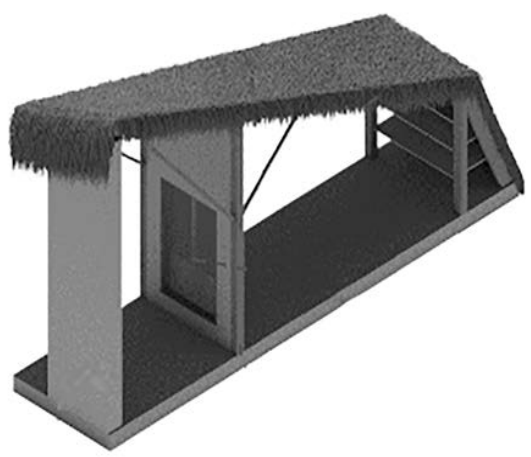

Figure 1: Modulation proposal - Project A

Through the making of the prototype (Figure 2), the student sought to study the stability of the structure subjected to a double inclination, in the longitudinal and transverse directions. The gantry was designed with auxiliary bars, and the process was directed towards the optimization of the structural solution, seeking the maximum inclination of the gantry to maintain its stability.

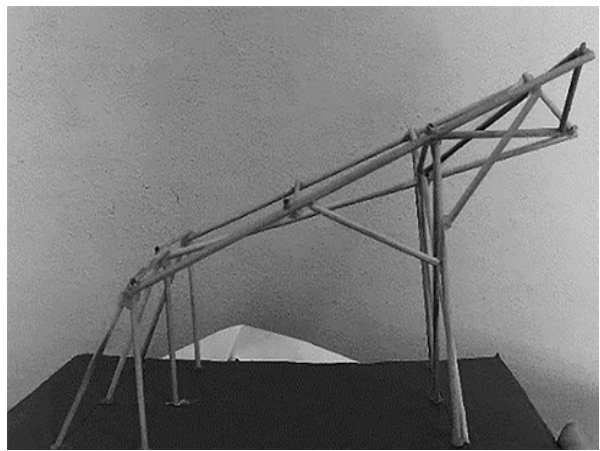

Figure 2: Structural prototype - Project A

The structural analysis was performed for the isolated frame (Figure 3 ) and for the combination of 8 frames. According to the student, the analysis of the set showed results of displacement of the set similar to that of the isolated frame, having demonstrated the stability of the proposed module. The cross sections chosen for the upper bar of the gantry, for the columns and for the secondary parts were validated, having a maximum utilization of $55 \%$. 

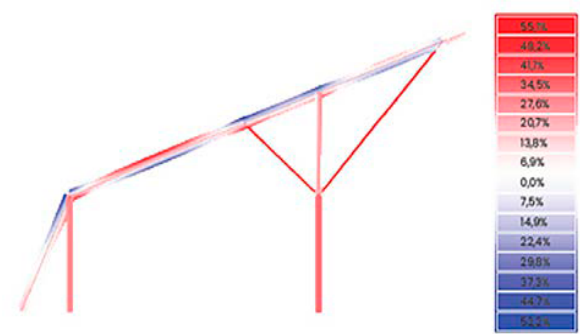

Figure 3: Computational structural analysis (graph of utilization of the selected profiles) - Project A

Project B was oriented towards the articulation of the space in such a way that it provided an internal patio for the practice of dance, as it is a sociocultural characteristic of the country. The spatial articulation for the creation of the internal patio would occur through the combination of classroom modules, these being sheltered by an independent cover with the objective of generating a shading in the common spaces and protection of the facades.

The structural design process developed by the student also aimed at validating and optimizing the initially designed structure. The making of the prototype (Figure 4) aimed to verify the stability of half of the structure, seeking to verify the maximum reach of the metal bars to generate structural balances, providing the maximum possible shading for the facades.

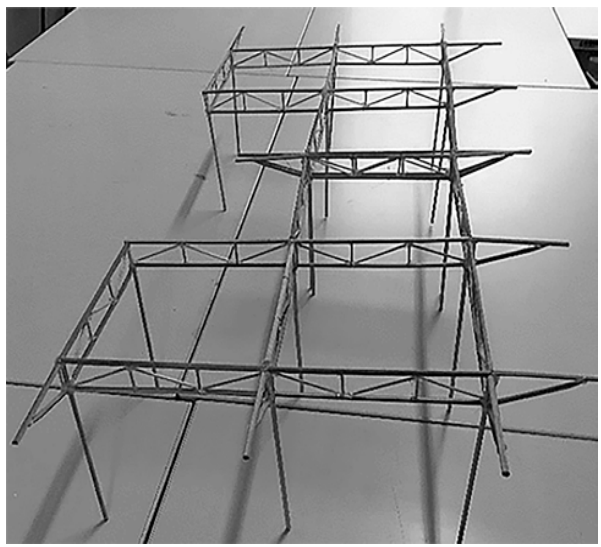

Figure 4: Structural prototype - Project B

The computational structural analysis had as its central aspect the verification of the buckling and displacement profiles. In the conversation with the engineer, the student was engaged in carrying out the verification by means of computational analysis and verification of the critical buckling load by the analytical method. The student said that he remembered having already had contact with the formulas used in the theoretical disciplines of structures, but that he would not have used them had they not been mentioned by the engineer.
The student reported that the structural analysis process enabled the structure to be reconfigured (Figure 5). Due to the dimension of the balance it was necessary to add internal bars at the ends of the truss in order to decrease its displacement. Regarding the internal part of the truss, the number of divisions was reduced in order to optimize the use of the bars. The new internal division of the truss resulted from the maximum support distance of the tiles and the attempt to optimize the use of the profiles. Still with the objective of increasing the use of the pieces, it was possible to reduce the number of columns, which had a direct impact on the proposed space articulation.

\section{Initial structure}

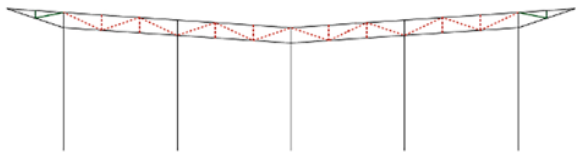

Final structure

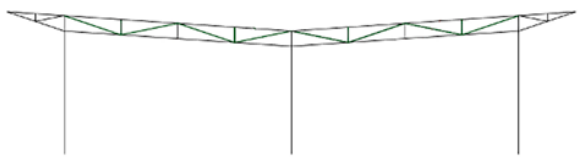

Figure 5: Initial and final structure - Project B

\section{RESULTS}

The projects developed by the students process a convergence between structural flexibility and spatial articulation, a way that the parametric design process enables a negotiation of spatial and material tests that occur in a circular manner, having an interaction and iteration in the process, corroborating the objective of the discipline. The structural analysis inserted in the same environment of the parametric modeling facilitated the process, allowing the instant visualization of the interactions performed with the structural performance, allowing the recursion in the analysis, synthesis and evaluation process to occur in an interactive and iterative way.

In questionnaires applied at the end of the course, a series of questions were asked related to the students' perception of the developed process and the computational tools used. When asked about the context, 10 (ten) students said they considered the project teaching environment to be very suitable for learning computational structural analysis, and the remaining 5 (five) considered it appropriate. Regarding the ease with which to learn the parametric process and the Grasshopper software, and the structural analysis using Karamba, the answers varied as follows: 


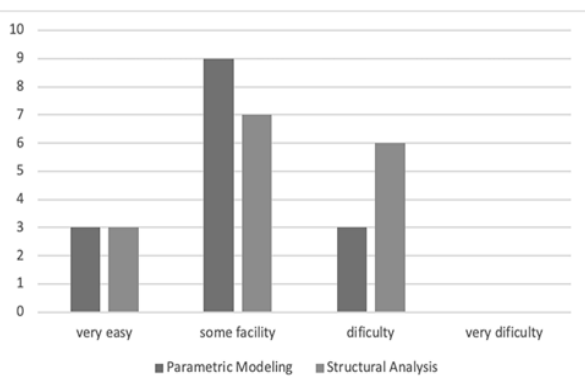

Figure 6: Students' perception of learning the parametric process and structural analysis

Regarding the participation of the structural engineer in the process, most of the answers ranged from very necessary (9 students) to necessary (4 students), with 2 (two) students considered the least necessary and 1 (one) student did not know how to answer, since he had not attended orientation classes with the engineer. The interesting thing about this answer is that some students who confirmed the need for the engineer to participate did not get to talk individually but felt that the presence of the engineer in the context validated the interdisciplinary process. The last question related to the students' perception asked if the parametric and structural analysis process had contributed to the spatial articulation of the developed project, with the answers varying as follows:

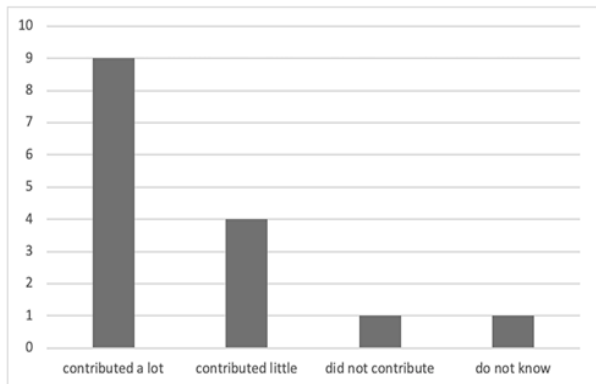

Figure 7: Students' perception of the process contribution to spatial articulation

Some students made comments regarding this question, with one student declaring that structural analysis helped to justify form, making the project "more complete, more authentic and consistent with reality". After assessing the perception of the process, students were asked whether they would take other subjects with the same format and should justify this answer. All students replied that they would very much like to take another course with this format, with a variety of justifications for this answer. Most emphasized the possibility of designing the space together with the structure, making it possible, according to one of the answers, "to arrive at a formal and structural result that would not be possible with other approaches". Other students highlighted the learning of structural computational analysis as a facilitator to develop the ability to think about the structure and design it more efficiently. And also, there were responses that highlighted the possibility generated by the process for its opening to potential dialogues.
In relationship to the concepts that would like to delve deeper into the next discipline, the unanimous response was to explore the parametric process. Some students pointed out that the structural analysis process linked to the parametric process was "very practical" and made it more "fluid", since "testing the solution was as easy as modeling it".

The man-machine-man conversation through parametric modeling was fundamental for the consolidation and circularity of the process. The parametric structural analysis software made it possible for recursion in the analysis, synthesis and evaluation process to occur in an interactive manner and without loss of information due to issues related to interoperability, allowing instantaneous visualization of the interactions performed with the structural performance. Conversation with the structural engineer was important not only for technical cooperation, but for developing a confidence in the structural solutions presented in the architectural design proposal. In addition, the conversation developed allowed the students to envision a relationship with the engineer different from the monologue relationships raised by current teaching.

With regard to the development of the structural analysis process, what was noted is that the difference between students with basic knowledge of structures and students with intermediate and advanced knowledge was not noticed, as seen in previous experiments, all of whom demonstrated the ability to perform model and evaluate the results of the analysis. The man-machine-man conversation was very important in this process, not only for the technical aspects, but also for the development of a new type of relationship with the structural engineer.

The diversity of the proposals presented demonstrated that the procedural orientation of the discipline was carried out, having been quite satisfactory for both the students and the teachers involved. However, one aspect was not possible to perform, presenting great potential for other experiments. During the process, some students requested that an integration be carried out with the materials laboratory or with an experimental construction site, demonstrating the desire to experiment with new materials, testing and experimenting with its possibilities of structural application. This process would allow the developed project to be guided by the developed material. This articulation was not possible, but it demonstrated that intuitively the process led them to a design reasoning oriented towards a material practice.

\section{DISCUSSION}

The experiment carried out through the discipline Structural Flexibility and Spatial Articulation was concerned with developing the three fundamental concepts for conducting the conversation in the project environment in digital culture: conducting the process through parametric modeling, performance simulation and orientation for tectonic aspects, which presented the potential to create a new language for the practice of design, more inclusive and dialectical, which can facilitate the exploration of solutions and include other agents in the design practice.

The experiment confirmed the potential of the parametric approach for teaching design beyond the use of the digital tool. By establishing socio-cultural parameters and structural and constructive parameters for the project, the students were able to actually work with the context, 
developing proposals oriented to the articulation of the space. The development of physical prototyping provided an awareness of the behavior of the proposed structure and served as a guideline for parametric modeling. The simulation of material performance using structural analysis software inserted in the parametric modeling environment facilitated interaction and iteration, providing a negotiation of parameters in order to promote structural flexibility in favor of the articulation of space. The information of the construction parameters was essential for the students to develop propositions with tectonic orientation, with lesser or greater intensity. Thus, we found that the experiment has pointed to a design method that admits the participation of other construction agents and future users, through design practices in which the architect conceives the design of the process more than the final product.

Practical-reflective teaching, centered on a conversational relationship between architects and engineers, can increase the complexity of the approach compared to the currently pedagogical practice. However, for this to occur, it is necessary to rethink architectural design teaching methods, seeking to develop processes open to the practice of conversation, encouraging interdisciplinary cooperation relations, oriented towards a dialectical practice. To enable a material dialectical practice, it is necessary to have an availability for an inversion of teaching procedures, in such a way that the material laboratories and the experimental site serve not only for the demonstration of concepts covered in expository classes, but that the classes displays serve to inform the experimentation carried out in the laboratories and on the jobsite. This allows the design processes developed by architecture students and architects to have a circularity and a recursion between material practice and digital practice, creating a connection between computer simulation and relations with the real world, covering not only material considerations, but also the social and political construction carried out through the project.

Digital tools incorporated into the design process have the potential to reconstruct the current model through the development of non-conservative practices, providing a common basis for the development of dialogic and dialectical relationships between architects and engineers. Thus, we believe that, as hybridizations in teaching, unlocking and merging some engineering and architecture technical codes, facilitated by digital tools, represent the degrees of freedom that allow a correct reconstruction of the technological system towards the construction of nonconservative processes for both professionals with a view to building democratic design processes. Only when the search breaks with the hegemony of values that will govern the technical codes and becomes possible the loss of alternative appropriations and even the creation of new codes, incorporating new sets of social values that can be technologically and socially sustained.

\section{ACKNOWLEDGMENTS}

I thank to my doctoral thesis advisor, professor Roberto Eustaáquio dos Santos, to engineer Guilherme Vaz and to all students who participated in the experiments carried out.

\section{REFERENCES}

Schön, D. (1987). Educating the reflective practitioner. San Francisco: Jossey-Bass, 1987.

Pask, G. (1976). Conversation Theory: applications in education and epistemology. Amsterdam: Elsevier.

Foerster, H. (2003). Ethics and second-order cybernetics. In Foerster, $H$. Understanding Understanding. Nova York: Springer-Verlag.

Dubberly, H.; Pangaro, P. What is Conversation? How can we design for effective conversation? Interactions Magazine, 16.4, $\quad$ p. 22, Retrieved from http://www.dubberly.com/articles/what-is-conversation.html.

Rebello, Y. (2000). Concepção Estrutural e a Arquitetura. São Paulo: Zigurate.

Black, G.; Duff, S. (1994). A Model for Teaching Structures: Finite Element Analysis in Architectural Education. Journal of Architectural Education, 48(1), 38-55. Retrieved from https://www.jstor.org/stable/1425308.

Kara, H. (2012) Redesigning Attitudes. In Kara, H; Georgoulias, A. (Ed.) Interdisciplinary Design: New Lessons from Architecture and Engineering (pp 10-15). Cambridge, Massachusetts: Harvard University Graduate School of Design.

Karamba. (2012) Karamba 3D: parametric engineering. Version 1.3.1. Viena: Clemens Preisinger e Bollinger und Grohmann ZT GmbH. Retrieved from https://www.karamba3d.com/download/.

Grasshoper. (2012) Grasshoper: algorithmic modeling for Rhino. Version 5.0. Nova York: David Rutten. Retrieved from http://www.rhino3d.com/download/grasshopper/1. 